\title{
Predicting and Measuring Surface Enlargement in Forward Rod Extrusion
}

Metal Forming Center of Excellence,

Atılım University,

Kızılcaşar Mahallesi,

06836 Incek, Gölbaşı,

Ankara, Turkey

e-mail: deniz.duran@atilim.edu.tr

\section{Izzet Ozdemir}

Assistant Professor

Department of Civil Engineering,

izmir Institute of Technology,

Gülbahçe Kampüsü,

Urla 35430, İzmir, Turkey

e-mail: izzetozdemir@iyte.edu.tr
Surface enlargement during bulk metal forming processes is one of the key parameters controlling the tribology at the tool-workpiece interface. Not only the surface roughness evolution but also the integrity of the lubricant layer critically reposes on surface enlargement. As an attempt to address this issue, in the first part of this work, a general, deformation gradient based surface enlargement description is implemented in a commercial finite element program. In the second part, forward rod extrusion tests with different area reductions are conducted using customized steel workpieces in which cylindrical copper rods are embedded through the depth. By sectioning the extruded parts and by identifying the position of the copper rods on the lateral surface, average surface enlargement values could be measured locally at different positions along the extrudate. Comparison of experiments and numerical predictions reveal that the deformation gradient based description performs reasonably well in capturing surface enlargement profiles both qualitatively and quantitatively. [DOI: 10.1115/1.4032261]

Keywords: surface enlargement, bulk metal forming, forward rod extrusion, finite element analysis (FEA)

\section{Introduction}

In bulk metal forming processes, workpieces are usually subjected to very high strains. Predictive modeling of these processes requires reliable material and interface characterization techniques. Ideally, characterization spectrum in terms of strain, strain rate, and temperature should cover the limits reached in the actual process. Similarly, characterization of the tool-workpiece interface requires consideration of contact pressure, yield stress of the material, sliding velocity, and temperature among other parameters.

There are two friction models widely used in the numerical modeling of forming processes. In the Coulomb model, frictional traction on the contacting surfaces is a fraction of contact pressure determined by the friction coefficient, $\mu$. In the constant shear stress model, frictional traction is obtained by multiplying the shear flow stress of the material by a friction factor, $\mathrm{m}$. Although they are extensively used in a wide range of applications, both of them have limitation as far as metal forming processes are concerned. For instance, in the Coulomb model, frictional traction increases linearly with increasing contact pressure. However, it is known that frictional traction cannot exceed the yield stress at pure shear. Although this limitation is captured by the constant shear stress model, it is insensitive to contact pressure, which can probably be considered as the most important parameter in manufacturing tribology. Furthermore, both of the aforementioned models are far from being satisfactory in reflecting the actual physical phenomenon taking place at the asperity level.

Asperity level resolution implies a distinction between the real and the apparent contact areas. Real contact area is the summation of areas of contacting asperities whereas apparent contact area ignores the fine scale surface topography over the contact area. Friction is highly dependent on the evolution of real contact area. In case of metal-metal contact, Bowden and Tabor [1] defines friction as "the force required to shear intermetallic junctions plus the force required to plough the surface of the softer metal by asperities on the harder surface." As can be deduced from this

Manuscript received May 31, 2015; final manuscript received November 30 , 2015; published online March 8, 2016. Assoc. Editor: Gracious Ngaile. definition, friction is dependent on two mechanisms, namely, adhesion (cold welding) of asperities (intermetallic junctions) and the ploughing effect. These mechanisms are strongly related with the surface topographies of the contacting bodies and the evolution of surfaces dictates the tribological behavior of tool-workpiece interface.

Surface enlargement is particularly critical in processes such as extrusion where the final product has a highly enlarged surface area as compared to the initial workpiece surface. Surface enlargement causes flattening of the asperities and increases frictional traction whereas surface contraction causes roughening of the asperities, which may lead to a decrease of the real contact area, thus overall friction coefficient. Moreover, surface enlargement is one of the most influential parameters for lubricant breakdown in cold extrusion since it affects the layer thicknesses of the tribological system significantly. There are various studies relating surface enlargement with lubricant thinning [2-6]. In Ref. [7], the authors claim that, apart from having a low friction coefficient, a good lubricant should possess enough coverability to spread over the enlarged surface during the process. According to Ref. [8], tribosystem fails when lubricant film thickness drops down below the value of the highest peak of tool asperities. It is anticipated in Ref. [2] that by simple measurement of surface enlargement, film weight distribution can be predicted in an indirect way. Furthermore, by establishing a relationship between surface enlargement and lubricant breakdown, different aspects of tool-workpiece interaction could potentially be identified, which in turn leads to improved process control.

Motivated by the preceding observations, a novel friction model incorporating surface evolution has been proposed, implemented, and used in predictive models of bulk forming operations in Refs. [9] and [10]. In this model, surface evolution is essentially described in terms of surface enlargement and contact pressure history. Surface enlargement is addressed both theoretically and algorithmically in a finite element based solution framework. The theoretical treatment and the derived expression for surface enlargement are based on an idealized forward rod extrusion process and its applicability under arbitrary deformations is questionable. Departing from this point, in this paper, a deformation gradient based surface enlargement description, which is valid 
under large arbitrary deformations and accompanying algorithmic framework are presented. The description is validated by a reference solution and an experimental measurement reported in the literature. Moreover, a novel experimental method enabling surface enlargement measurements in forward rod extrusion process is developed and realized for different area reductions. These experimental results are used to assess the capabilities of the proposed surface enlargement description.

The paper is organized as follows. In Sec. 2, the description of surface enlargement under arbitrary deformations is presented. Afterward, the proposed scheme is critically assessed using a benchmark problem with a reference solution and an experimental result from literature. Sections 4 and 5 are reserved for the details of experimental setup and finite element analysis (FEA), respectively. Comparison between experimental measurements and numerical predictions is presented in Sec. 6 before the paper is closed by the Summary and Outlook section.

\section{Surface Enlargement Under Arbitrary Deformations}

For certain idealized processes such as upsetting, under certain assumptions, e.g., volume constancy and negligible friction, it is possible to calculate surface enlargement $(\psi)$ analytically as exemplified in Ref. [9]. This reference focuses on frictionless axisymmetric forward rod extrusion and proposes a strain based expression for surface enlargement given as

$$
\psi \cong 1+\varepsilon_{r}+\varepsilon_{z}
$$

where $\varepsilon_{r}$ and $\varepsilon_{z}$ are radial and axial true normal strains, respectively. However, the validity of this expression under arbitrary deformations, e.g., nonaxisymmetric forming processes or more complex processes such as backward can extrusion, does not hold anymore.

In order to have a general expression for surface enlargement, an alternative method is used here, which is based on mapping the unit material vectors by the deformation gradient. In Fig. 1, within a geometrically nonlinear framework, a deformable body is shown in undeformed and deformed configurations, respectively. An elemental area $\mathrm{dA}$ in the undeformed configuration becomes da upon deformation. In order to relate these two areas, two different paths can be followed.

In the first method, called as Lagrangian approach, material unit vectors in undeformed state are mapped by the deformation gradient tensor $\mathbf{F}$. In a 2D setting, two orthogonal material unit

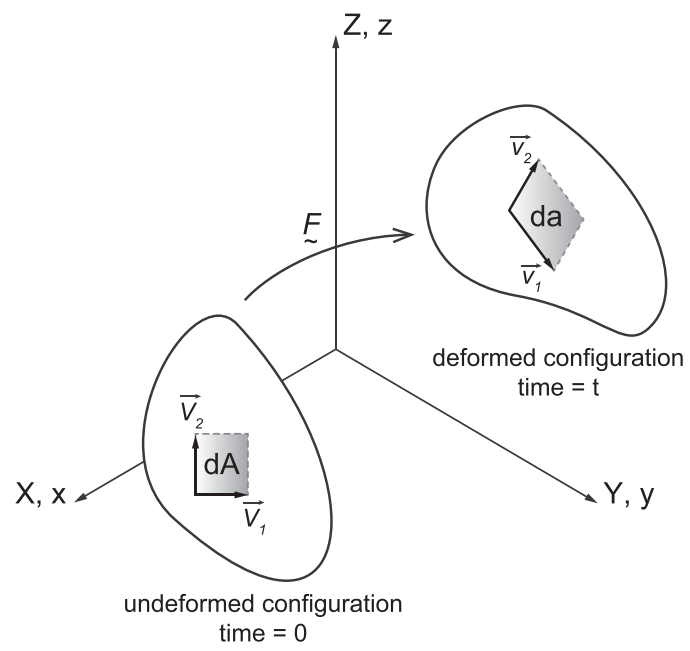

Fig. 1 A deformable body in undeformed and deformed state. Material vectors are mapped by the deformation gradient tensor $F$. vectors are considered as shown in Fig. 1. The magnitude of cross-product of these vectors is equal to the shaded unit surface area. Each of these vectors are mapped through

$$
\begin{aligned}
& \mathbf{v}_{1}=\boldsymbol{F} \mathbf{V}_{1} \\
& \mathbf{v}_{2}=\boldsymbol{F} \mathbf{V}_{2}
\end{aligned}
$$

and the magnitude of cross product $\mathbf{v}_{1} \times \mathbf{v}_{2}$ yields the current surface area. Then, the surface enlargement can be calculated simply as $\psi=$ da.

In the second method, called as Eulerian approach, orthogonal material unit vectors $\mathbf{q}_{1}$ and $\mathbf{q}_{2}$ in the deformed configuration are taken as the starting point. These two vectors are mapped by the inverse of the deformation gradient tensor in order to find their state in the undeformed configuration, i.e., $\mathbf{Q}_{1}=\boldsymbol{F}^{-1} \mathbf{q}_{1}$ and $\mathbf{Q}_{2}=\boldsymbol{F}^{-1} \mathbf{q}_{2}$. The elemental area as calculated from the cross product of $\mathbf{Q}_{1}$ and $\mathbf{Q}_{2}$ is used to quantify the surface enlargement via $\psi=1 / d$ A, where $d A=\left\|\mathbf{Q}_{1} \times \mathbf{Q}_{2}\right\|$.

Aforestated two methods are in fact alternative forms of Nanson's formula well known in continuum mechanics [11]. In the rest of the paper, Lagrangian approach is preferred. A comparison between these two approaches is given in Ref. [12].

For an axisymmetric analysis as shown in Fig. 2, one of the material unit vectors (called as normal unit vector) is perpendicular to the investigated plane and expressed as

$$
\mathbf{N}=-\mathbf{e}_{\theta}
$$

at each point on the outline. The other material unit vector (called as tangential unit vector) lies in the investigated plane and expressed as

$$
\mathbf{T}=T_{r} \mathbf{e}_{r}+T_{z} \mathbf{e}_{z}
$$

where $\mathbf{e}_{\theta}, \mathbf{e}_{r}$, and $\mathbf{e}_{z}$ are the unit vectors in cylindrical polar coordinates axes.

Considering a discretization with four-noded quadrilateral elements, first the components of the tangential vector are calculated from the nodal coordinates for each outline edge. Afterward, a nodal averaging is done and calculated unit vectors are assigned to the nodes.

The remaining key component of the formulation is the calculation of the deformation gradient, which is expressed as

$$
\boldsymbol{F}=\frac{\partial \mathbf{x}}{\partial \mathbf{X}}=\boldsymbol{I}+\frac{\partial \mathbf{u}}{\partial \mathbf{X}}
$$

where $\mathbf{X}, \mathbf{x}$, and $\mathbf{u}$ are the undeformed position vector, deformed position vector, and displacement vector of a material point, respectively. $\boldsymbol{I}$ is the second order identity tensor. In cylindrical

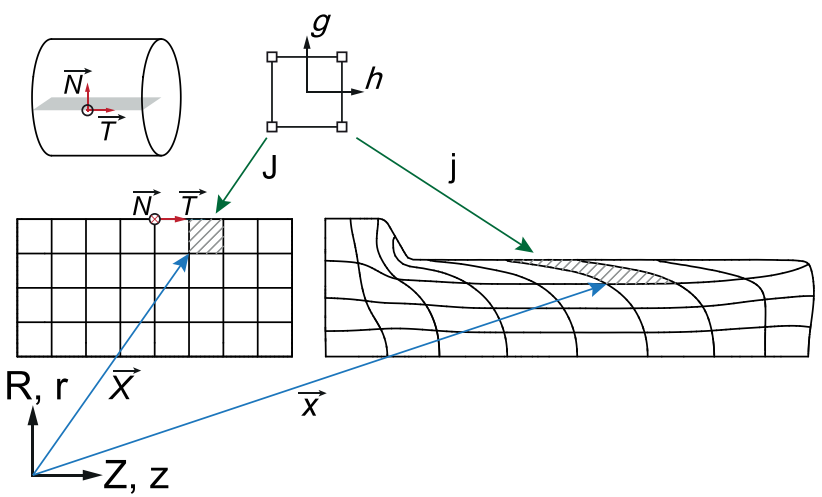

Fig. 2 Discretization of an axisymmetric component by fournode quadrilaterals 
polar coordinates (see, e.g., Ref. [13] for details), for an axisymmetric body, the components of the deformation gradient tensor take the following explicit form:

$$
\underline{\boldsymbol{F}}=\left[\begin{array}{ccc}
1+\frac{\partial u_{r}}{\partial R} & 0 & \frac{\partial u_{r}}{\partial Z} \\
0 & 1+\frac{u_{r}}{R} & 0 \\
\frac{\partial u_{z}}{\partial R} & 0 & 1+\frac{\partial u_{z}}{\partial Z}
\end{array}\right]
$$

where $u_{r}$ and $u_{z}$ are the components of the displacement vector of a material point which was located at $(R, Z)$ in the undeformed configuration. In order to calculate $\partial u_{r} / \partial R, \partial u_{r} / \partial Z, \partial u_{z} / \partial R$, and $\partial u_{z} / \partial Z$, the isoparametric mapping concept is used to express the gradient as

$$
\left[\begin{array}{ll}
\frac{\partial u_{r}}{\partial R} & \frac{\partial u_{r}}{\partial Z} \\
\frac{\partial u_{z}}{\partial R} & \frac{\partial u_{z}}{\partial Z}
\end{array}\right]=\left[\begin{array}{ll}
\frac{\partial u_{r}}{\partial g} & \frac{\partial u_{r}}{\partial h} \\
\frac{\partial u_{z}}{\partial g} & \frac{\partial u_{z}}{\partial h}
\end{array}\right]\left[\begin{array}{ll}
\frac{\partial g}{\partial R} & \frac{\partial g}{\partial Z} \\
\frac{\partial h}{\partial R} & \frac{\partial h}{\partial Z}
\end{array}\right]=\underline{G}_{1} \underline{G}_{2}
$$

through the chain rule. The first term on the right hand side is the gradient of the displacement vector with respect to natural coordinates $g$ and $h$ of the parent element and straight forward to calculate through the interpolations defined as

$$
u_{r}=\sum_{i=1}^{n_{\text {node }}} N_{i}(g, h)\left(u_{r}\right)_{i}, \quad u_{z}=\sum_{i=1}^{n_{\text {node }}} N_{i}(g, h)\left(u_{z}\right)_{i}
$$

for an element with $n_{\text {node }}$ nodes. $\left(u_{r}\right)_{i}$ and $\left(u_{z}\right)_{i}$ are the displacement components of node $i$ and $N_{i}(g, h)$ is the shape function associated with node $i$. Isoparametric formulation enforces the geometric mapping between points of the parent element and physical element through the following interpolations (which are identical to interpolation used in Eq. (9)

$$
R=\sum_{i=1}^{n_{\text {node }}} N_{i}(g, h) R_{i}, \quad Z=\sum_{i=1}^{n_{\text {node }}} N_{i}(g, h) Z_{i}
$$

where $R_{i}$ and $Z_{i}$ are the undeformed coordinates of node $i$. The Jacobian of this mapping which is defined as

$$
\underline{J}=\left[\begin{array}{ll}
\frac{\partial R}{\partial g} & \frac{\partial R}{\partial h} \\
\frac{\partial Z}{\partial g} & \frac{\partial Z}{\partial h}
\end{array}\right]
$$

can be calculated using Eq. (10). In fact, the inverse of $J$ is the second term in Eq. (8) ( $\underline{G}_{2}$ matrix), which can be verified by checking the identity $\underline{J} \underline{G}_{2}=\underline{I}$.

Once the unit vectors and the deformation gradient are available, mapped material vectors $\mathbf{v}_{1}$ and $\mathbf{v}_{2}$ are calculated. Finally, the magnitude of cross product $\mathbf{v}_{1} \times \mathbf{v}_{2}$ yields the sought surface enlargement/contraction.

Due to large strains, the Lagrangian meshes used in FEA of bulk metal forming processes are prone to excessive mesh distortion. In dedicated FEA tools, this problem is addressed by remeshing algorithms, where a new mesh is generated by maintaining the outline of the old distorted mesh. Since a new mesh is generated, element nodal connectivities and node numbers change. These result in a loss of history of user field variables, such as material unit vectors in the current case. In order to overcome this issue, a 1D mapping algorithm is implemented, which is capable of transferring the history of the surface variables between consecutive increments when a remeshing takes place. The correspondence between the newly created node and the edge of the previous mesh (the edge within which the newly created node falls) has to be constructed. Once this is done, the history variable of the newly created node is calculated through a simple linear interpolation using the nodal values of the edge.

The algorithmic framework is implemented in the commercial FE software Marc Mentat. In Sec. 3, the formulation and the algorithm is validated using two examples.

\section{Validation Cases}

Two validation cases are considered in this section. The first one has an accurate reference solution and the second one is, to the knowledge of the authors, the only experimental results reported in the literature.

3.1 Truncated Cone. In this example, surface enlargement on the lateral surface of a truncated cone is investigated. Top and bottom surfaces of the truncated cone are confined by two fixed curves (rigid tool), and the lateral surface is deformed by rotating the rigid tool leaning against it, see the sketches on the top left corner of Fig. 3. It has to be noted that the deformation considered in this example is not realistic and designed for the proof of concept. An axisymmetric model is used whose initial geometry is shown in Fig. 3. A clockwise rotation of $22 \mathrm{deg}$ around the shown axis of rotation is imposed on the rigid tool without friction in between. The reference solution for surface enlargement of the lateral surface can be derived using the formula for the lateral area of a truncated cone

$$
A_{\text {lateral }}=\pi(R+r) s
$$

where $s$ in the slant height, $R$ and $r$ are the larger and the smaller radii of the truncated cone, respectively. This equation can be adapted for a slice of slant height $\Delta s$ as

$$
A_{\text {slice }}=\pi\left(R_{s}+r_{s}\right) \Delta s
$$

where $R_{s}$ and $r_{s}$ are the corresponding radii of the slice. In the limit as $\Delta s$ approaches to zero, an exact surface enlargement profile along the slant of a truncated cone can be reached. Since final radii profile along the slant height is prescribed by the rotating tool motion, area of a slice in the deformed configuration can be calculated. For a tool rotation of $22 \mathrm{deg}$, the larger radius of the truncated cone reduces to $34.789 \mathrm{~mm}$ whereas the smaller radii and the height stay the same. Using these deformed radii values of the truncated cone and the number of slices, the radii of a slice can be determined and the deformed area can be calculated using Eq. (13). Similarly, plugging the undeformed radii and slant height of the slice into Eq. (13) yields the undeformed area of the corresponding slice. With these two values in hand, surface enlargement can be determined for a particular slice. Through some numerical examples (by writing a small function in MATLAB based on Eq. (13)), it has been seen that for this particular case, setting $\Delta s$ smaller than $s / 1000$ makes negligible changes in the calculated surface area profile. Therefore, the profile obtained by using $\Delta s=s / 1000$ is taken as the accurate reference solution.

In Fig. 3, reference curve is compared with the strain based and the deformation gradient based surface enlargement results. The label strain based refers to Eq. (1) proposed by [9] and the label deformation gradient based implies the proposed description in this paper. As seen from the figure on the right hand side of Fig. 3 , the deformation gradient based results are almost coincident with the reference curve, whereas the strain based results deviate from the reference solution up to $9 \%$.

3.2 Backward Can Extrusion. Experimental identification of surface enlargement is a complicated task due to extreme straining of the surface. In this field, the only experimental study to the knowledge of the authors is presented in Ref. [14]; where 

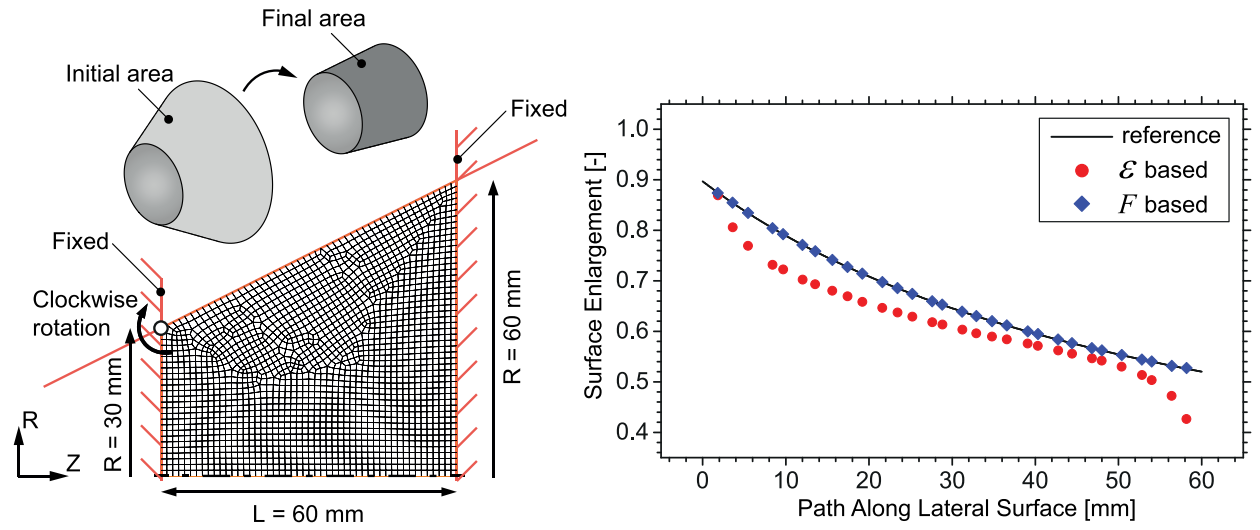

Fig. 3 Geometry of the truncated cone and comparison between analytical solution and numerical predictions

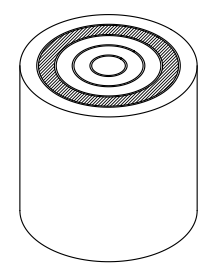

before extrusion

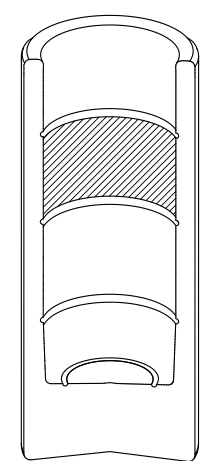

after extrusion

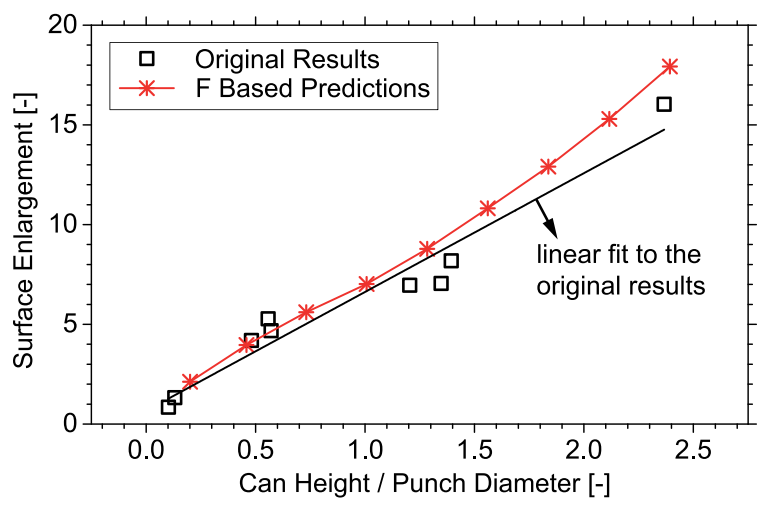

Fig. 4 Surface enlargement in backward can extrusion [14] and comparison between experimental and predicted surface enlargement values

surface enlargement in backward can extrusion process was measured. To this end, concentric grooves were milled on the end face of the cylindrical billet, as schematically shown in Fig. 4. After deformation, grooves immigrate to the inner surface of the extruded can. The ratio of the deformed area confined by two consecutive grooves and the corresponding undeformed area (shown as shaded areas in undeformed and deformed geometries, respectively) yields the average surface enlargement.

The proposed groove based measurement technique is applied in a backward can extrusion process with an ideal equivalent plastic strain of 0.7 (corresponds to $\Delta A / A_{0}=0.5$, where $\Delta A$ is the reduction in cross-sectional area and $A_{0}$ is the initial crosssectional area of the billet). However, due to severe surface enlargement, grooves are heavily stretched and disappeared. Only a slight mark is observed on the inner surface of extruded cans, which makes the determination of surface enlargement almost impossible. On the other hand, all the necessary information to simulate the process is available in Ref. [14]. Flow curve is given as

$$
\sigma_{f}=652 \varepsilon_{\mathrm{pl}}^{0.22}
$$

and shear friction model with a friction factor of 0.08 is used. Punch geometry is reproduced using the technical drawing supplied in the same reference. Thermal effects are neglected and a punch stroke of $10 \mathrm{~mm}$ is imposed. Surface enlargement values along the can height are calculated using the deformation gradient based description and compared with the experimental results as shown on the right hand side of Fig. 4 (experimental results are read from the reference by means of a digitizer).
Especially at relatively low can height/punch diameter ratios, there is agreement between the measurements and the predictions. However, it has to be noted that as the stroke increases, the deviation reaches up to $12 \%$.

\section{Experimental Study}

In this section, a method for the experimental determination of average surface enlargement is presented. The method is inspired by the visioplasticity study detailed in Ref. [15]. In this reference, dynamic microstructure evolution of aluminum during hot forward extrusion process was investigated and by means of visioplasticity, the material flow predicted by FEA was validated. The same method is utilized in the current study, yet aiming at the measurement of surface enlargement/contraction.

Surface enlargement is evaluated for cold forward rod extrusion process with three different area reductions as shown in Table 1. In the rest of the paper, these area reductions are called by corresponding ideal equivalent plastic strain values. It has to be noted that although a single value of equivalent plastic strain is used to label each case, plastic strain distribution within the extruded billets are nonuniform. Three repetitive tests are done for each process. For all cases, the initial workpiece diameter is $25 \mathrm{~mm}$ and the cone angle is $2 \alpha=120 \mathrm{deg}$. $16 \mathrm{MnCr} 5$ steel is used as workpiece material and workpieces are phosphate coated and lubricated with soap prior to extrusion. Holes with a diameter of $1 \mathrm{~mm}$ are drilled all through the transverse direction by using small hole drilling electrical discharge machining (EDM) machine. This machine has a numerical controlled table which ensures precise positioning of the holes. Since surface enlargement of the 
Table 1 Geometric details of the conducted extrusion tests

\begin{tabular}{llc}
\hline \hline $\begin{array}{l}\text { Exit diameter } \\
\left(D_{f}, \mathrm{~mm}\right)\end{array}$ & $\begin{array}{c}\text { Plastic strain } \\
\left(\varphi=\ln \frac{A_{0}}{A_{f}}\right)\end{array}$ & Relative area \\
\hline 17.62 & 0.7 & \\
15.16 & 1.0 & 75 \\
12.41 & 1.4 & 50 \\
\hline
\end{tabular}
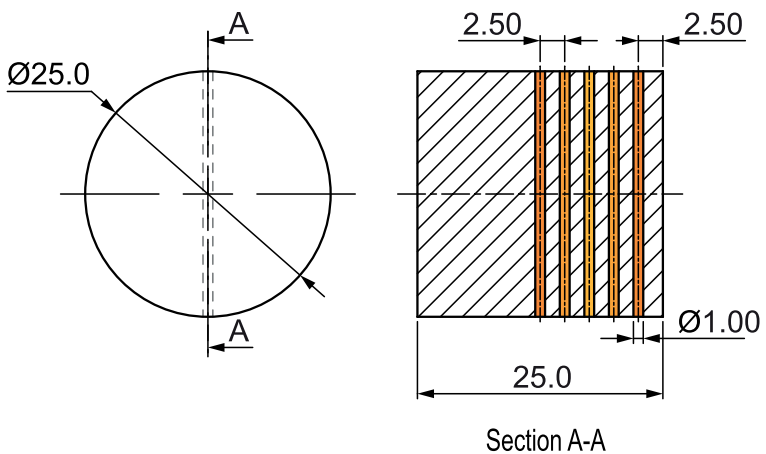

Fig. 5 Workpiece geometry with copper pins

extrudate is the primary concern, holes span half of the workpiece (lengthwise) as shown in Fig. 5.

Holes are equally spaced by a distance of $2.5 \mathrm{~mm} .99 .9 \%$ purity copper is used as pin material because of its low flow stress and high formability. $25 \mathrm{~mm}$ long pins of $1 \mathrm{~mm}$ diameter are prepared using wire EDM and pushed into the drilled holes through the workpiece. For each workpiece, five copper pins are assembled. Since the workpiece diameter and pins length are equal to $25 \mathrm{~mm}$, no secondary operation like grinding or sandpapering is necessary after the assembly operation.

In Fig. 6, undeformed and deformed areas are shown schematically. The coincidence points between the copper pins and the lateral cylindrical surface of the workpiece defines an area. In the initial state, the undeformed area is calculated by

$$
A_{0}=\pi D_{0} L_{0}
$$

where $L_{0}$ and $D_{0}$ corresponds to $1.5 \mathrm{~mm}$ and $25 \mathrm{~mm}$, respectively. After extrusion, the enlarged area is obtained by using

$$
A_{f}=\pi D_{f} L_{f}
$$

where $D_{f}$ is the exit diameter of the die, i.e., the extrudate diameter and $L_{f}$ is the deformed length between the coincidence points. With these quantities in hand, average surface enlargement for each area can be calculated simply by

$$
\psi=\frac{D_{f} L_{f}}{D_{0} L_{0}}
$$

Extruded parts are sectioned from the midplane by wire EDM to reveal copper lines. Following that, polishing is applied and macrophotographs are shot together with calibrated length bars. The locations where copper lines coincide with the surface are detected by image processing.

\section{Finite Element Analysis}

In order to get reliable predictions for surface enlargement through FEA, bulk material characterization spanning the complete strain, strain rate, and temperature spectrum of the actual process is essential. An in depth study of material characterization of $16 \mathrm{MnCr} 5$ steel by using a deformation dilatometer is presented

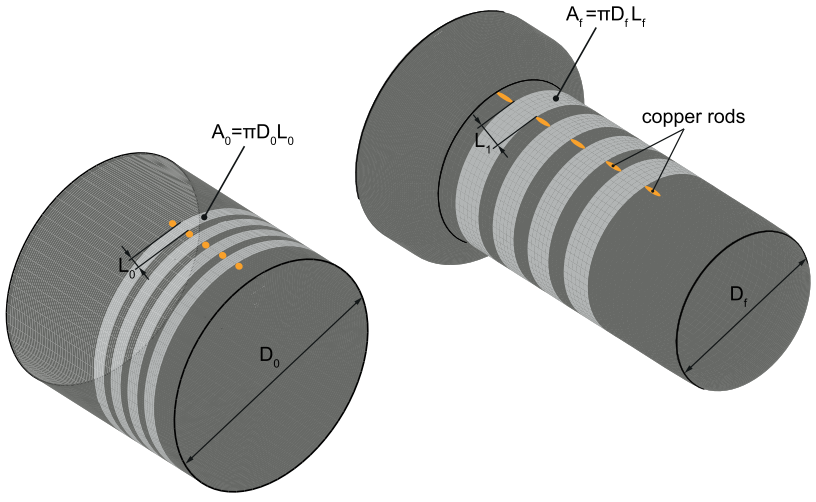

Fig. 6 Enlargement of defined areas in forward rod extrusion

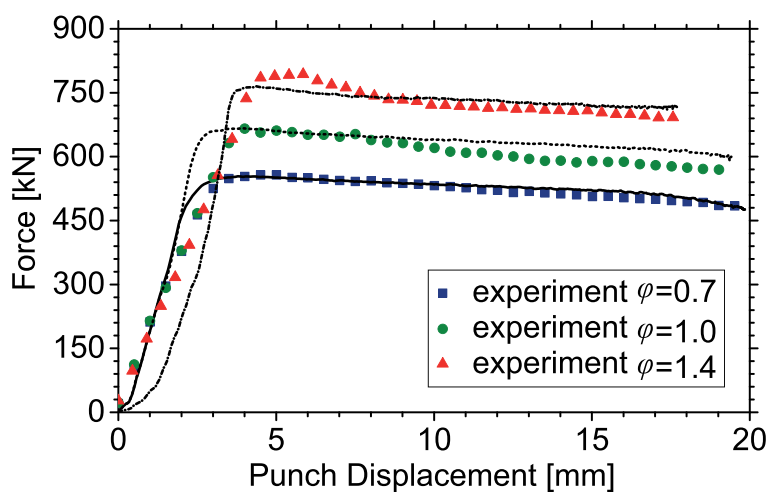

Fig. 7 Force-displacement curves

in Ref. [12] with a special focus on potential pitfalls and remedies, particularly at high strain rates.

Since friction substantially affects surface evolution, a good friction characterization should be conducted as well. For this purpose, experimental force-displacement curves are taken as reference and force-displacement curves calculated by FEA are scaled by varying friction factor until a satisfactory agreement is obtained. General friction model developed by Wannheim and Bay is used in FEA since it yields more accurate results in bulk forming processes, see Ref. [16]. By employing this procedure, friction factors are found as $0.3,0.25$, and 0.2 for $0.7,1.0$, and 1.4 ideal equivalent plastic strains, respectively. Because of the increase in overall contact pressure with increasing area reduction, a unique friction factor could not be obtained covering all three processes. Experimental and FEA based force-displacement curves are shown in Fig. 7.

\section{Results and Discussion}

Marc Mentat has the capability of tracking material flow according to a preset pattern. Copper pins are defined as the initial pattern and their final layout as predicted by FEA are compared with macrophotos of sectioned specimens in Fig. 8. For $\varphi=0.7$ and $\varphi=1.0$, all five copper pins reached the die exit. However, for $\varphi=1.4$, due to the fact that the extrudate length is the largest and the stroke is limited by the die configuration, the last copper pin could not reach the extrudate surface. As can be seen in Fig. 8, the experimental and the predicted locations where copper pins coincide with the extrudate surface are in agreement. However, as the deformation gets more severe, a deviation is observed on the symmetry axis of the workpieces, especially for $\varphi=1.0$ and $\varphi=1.4$. This deviation might be due to the difference between flow characteristics of copper and steel. Furthermore, for $\varphi=0.7$ and $\varphi=1.0$; separation of copper pins from the bulk in the neighborhood of the 


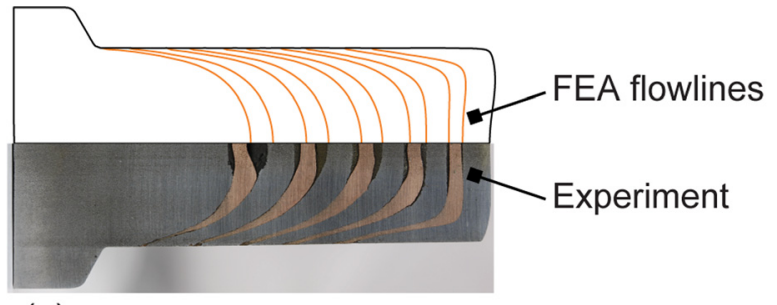

(a)

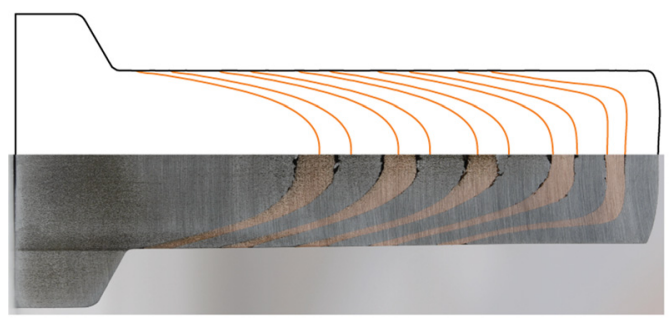

(b)

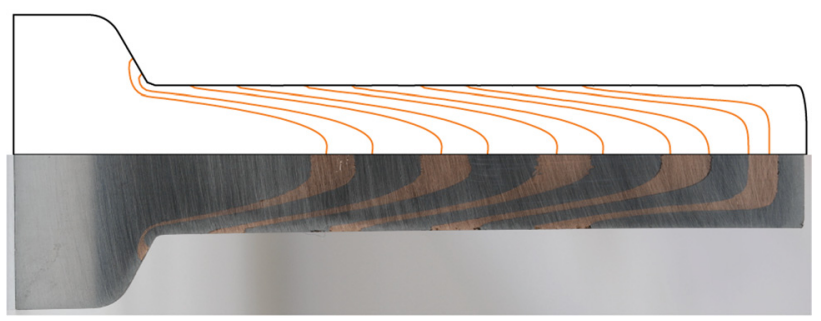

(c)

Fig. 8 Comparison between experiments and FEA predictions of flowlines (a) $\varphi=0.7$, (b) $\varphi=1.0$, and (c) $\varphi=1.4$

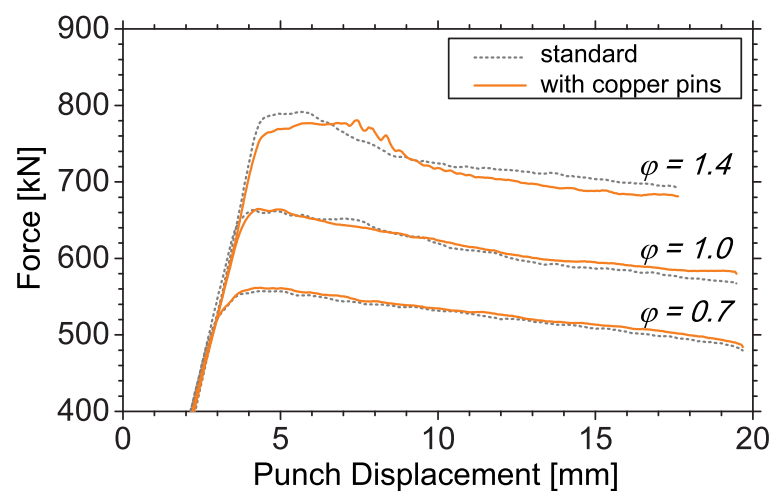

Fig. 9 Force-displacement curves with standard and modified workpieces

symmetry axis is clearly visible. Such separations disappear for $\varphi$ $=1.4$ probably due to relatively large pressures developing at the center of the workpiece.

In order to assess the influence of copper pins on measured responses, processes are repeated using standard specimens (without copper pins). A comparison of force-displacement curves are shown in Fig. 9. Curves corresponding to standard specimens are the mean of 15 tests; whereas the curves of customized specimens are the mean of three repetitions for each case. Apart from the slight difference in trend and abrupt chatter in postpeak region for $\varphi=1.4$, the curves are very close. The reason for postpeak chatter for $\varphi=1.4$ might be due to excessive compression of the weaker copper pins which reveals itself as successive valleys in the postpeak chatter zone. The reason for this peculiar behavior has to be investigated thoroughly.

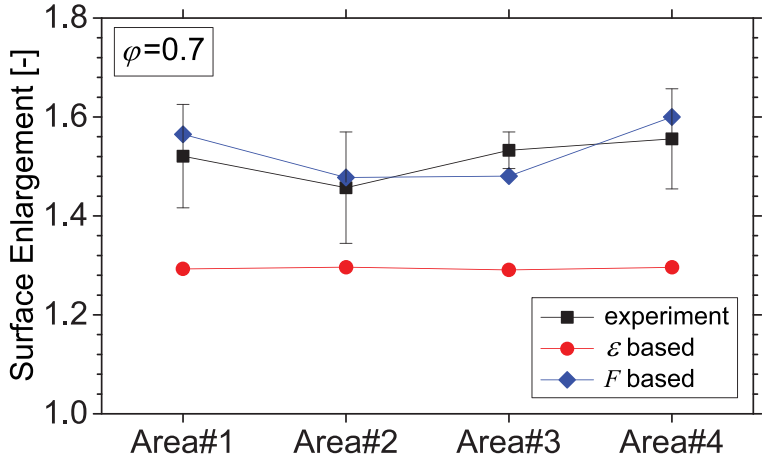

Fig. 10 Experimental versus predicted surface enlargement values for $\varphi=0.7$

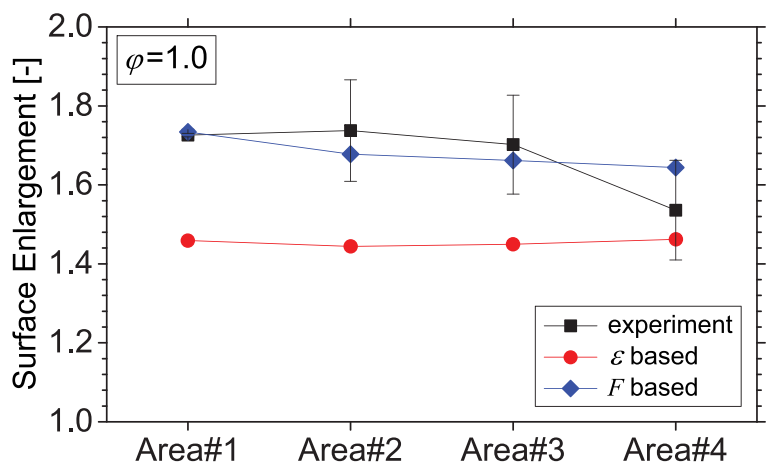

Fig. 11 Experimental versus predicted surface enlargement values for $\varphi=1.0$

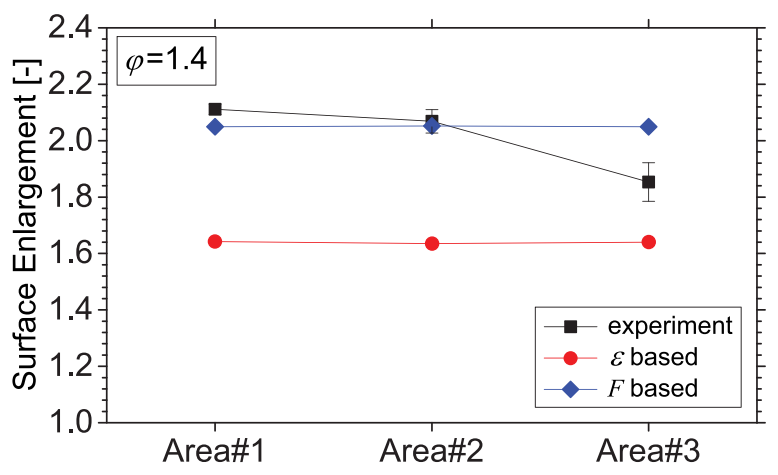

Fig. 12 Experimental versus predicted surface enlargement values for $\varphi=1.4$

Copper rods define boundaries of the areas which are used to measure the average surface enlargement/contraction. The locations where copper rods coincide with extrudate lateral surface are projected on FE mesh. The predicted surface enlargement values available at nodes are used to calculate an average surface enlargement value corresponding to each interval defined by these projection points. Experimental values are compared with the strain and deformation gradient based formulation results as shown in Figs. $10(\varphi=0.7), 11(\varphi=1.0)$, and $12(\varphi=1.4)$, respectively.

Areas are numbered from 1 to 4 with respect to material flow direction. The area closest to extrusion butt is referred to as Area $\# 1$ and the area closest to extrudate tip is referred to as Area \#4. Experimental results show moderate repeatability. Maximum standard deviation between the measured coordinates is not more 
than $0.15 \mathrm{~mm}$. Agreement between the deformation gradient based formulation and experimental results are considerably good. The strain based predictions deviate from the experimental results around $15 \%$ for $\varphi=0.7$ and $\varphi=1.0$; and the deviation increases up to $20 \%$ for $\varphi=1.4$. It has to be noted that deformation gradient based formulation overshoots the experimental result at Area $\# 3$ for $\varphi=1.4$

\section{Summary and Outlook}

In this paper, surface enlargement in large deformation processes is investigated both numerically and experimentally. In the numerical part of the study, a deformation gradient based surface enlargement description ( $F$ based) is shown, which is valid under arbitrary deformations. Then, this description is implemented into a FEA framework by employing user subroutines. In order to validate the implementation, two different cases are considered; one of which has a relatively simple geometry and well defined boundary conditions and the other is based on reproducing the experimental results reported in Ref. [14]. Both cases reveal that the proposed description holds, moreover performs better than an existing description ( $\varepsilon$ based).

In the experimental part of the study, cold forward rod extrusion processes with three different area reductions are realized. A novel method is proposed where average surface enlargement values are determined along extrudate surfaces by using customized workpieces. To this end, copper pins are embedded in steel workpieces in order to create predefined areas on the surface. Enlargement of these areas are monitored upon deformation by sectioning the extruded parts and then tracing the coincidence points of copper pins with the surface. By means of thorough material characterization and proper friction factor selection, these processes could be simulated accurately; so that experimental and predicted surface enlargement values could be compared. Implemented description ( $F$ based) is found to be successful in capturing surface enlargement both qualitatively and quantitatively; whereas deviations up to $20 \%$ are observed with the existing description ( $\varepsilon$ based). Furthermore, force-displacement curves are in agreement when both standard and customized workpieces are used which indirectly indicates that the material flow behavior is affected up to an admissible extent and that the experimental method can be used safely in determining average surface enlargement.

Since the implemented surface enlargement description is valid under arbitrary deformations, it can be used in any type of forming processes such as die forging, rolling, and deep drawing. In connection with the tribological behavior of these processes, there is an increasing interest in physics based models, which attempt also to take the effect of surface enlargement/contraction into account. It is the authors' opinion that the implemented and validated surface enlargement description would be particularly useful in such friction models. Extension of the method to threedimensional problems is doable, but the implementation requires robust book keeping and mapping algorithms due to remeshing of evolving 3D geometries.

Using the same experimental technique proposed in this study, it is possible to determine surface enlargement in other basic cold forging processes in a straightforward manner; such as backward can extrusion in which surface enlargement is known to reach much higher values. However, attention must be paid for complex workpiece geometries (e.g., die forging) where correct interpretation of the outline is critical.

\section{References}

[1] Bowden, F. P., and Tabor, D., 1982, Friction-An Introduction to Tribology, Robert E. Krieger Publishing Company, Malabar, FL.

[2] Saiki, H., Ngaile, G., and Ruan, L., 1997, "Influence of Die Geometry on the Workability of Conversion Coatings Combined With Soap Lubricant in Cold Forming of Steels," J. Mater. Process. Technol., 63(1-3), pp. 238-243.

[3] Saiki, H., and Marumo, Y., 2003, "Influence of the Roughness Geometry of Tool Surface and the Flow Stress of Coated Solid Lubricants on TriboConditions in Cold Forging," J. Mater. Process. Technol., 140(1-3), pp. 25-29.

[4] Ruan, L., Saiki, H., Marumo, Y., and Imamura, Y., 2005, "Evaluation of Coating-Based Lubricants for Cold Forging Using the Localised Rod-Drawing Test," Wear, 259(7-9), pp. 1117-1122.

[5] Ngaile, G., Saiki, H., Ruan, L., and Marumo, Y., 2007, “A Tribotesting Method for High Performance Cold Forging Lubricants," Wear, 262(5-6), pp. 684-692.

[6] Lee, H. Y., Noh, J. H., and Hwang, B. B., 2013, "Surface Stresses and Flow Modes on Contact Surface in a Combined Double Cup Extrusion Process," Tribol. Int., 64, pp. 215-224.

[7] Goto, Y., Wakasugi, S, and Kozai, T., 1982, "A Test for Investigating the Lubrication Properties of Solid Lubricants in Cold Metal Forming," J. Mech Work. Technol., 6(1), pp. 51-62.

[8] Bay, N., Lassen, S., Pedersen, K. B., Maegaard, V., and Wanheim, T., 1991 , "Lubrication Limits in Backward Can Extrusion at Low Reductions," CIRP Ann.-Manuf. Technol., 40(1), pp. 239-242.

[9] Stahlmann, J., Nicodemus, E. R., Sharma, S. C., and Groche, P., 2012, "Surface Roughness Evolution in FEA Simulations of Bulk Metal Forming Process," Wear, 2008, pp. 78-87.

[10] Ludwig, M., Stahlmann, J., and Groche, P., 2012, "Advanced Friction Model for Cold Forging Processes," 14th International Conference on Metal Forming, Krakow, Poland, pp. 1003-1006.

[11] Wriggers, P., 2008, Nonlinear Finite Element Methods, Springer-Verlag, Berlin, Heidelberg.

[12] Duran, D., 2014, “Analysis of Cold Extrusion,” M.Sc. thesis, Atılım University, Ankara, Turkey.

[13] Bower, A. F., 2012, “Applied Mechanics of Solids," http://solidmechanics.org

[14] Bay, N., Wibom, O., and Nielsen, J. A., 1995, "A New Friction and Lubrication Test for Cold Forging," CIRP Ann.-Manuf. Technol., 44(1), pp. 217-221.

[15] Güzel, A., Jäger, A., Parvizian, F., Lambers, H.-G., Tekkaya, A. E., Svendsen, B., and Maier, H. J., 2006, "A New Method for Determining Dynamic Grain Structure Evolution During Hot Aluminum Extrusion," J. Mater. Process. Technol., 212(1), pp. 323-330.

[16] Bay, N., 1987, "Friction Stress and Normal Stress in Bulk Metal-Forming Processes," J. Mech. Work. Technol., 14(2), pp. 203-223. 